\title{
On Scheduling Policies for Streams of Structured Jobs ${ }^{\star}$
}

\author{
Aldric Degorre, Oded Maler \\ VERIMAG-UJF-CNRS, 2 av. de Vignate, 38610 Gières, France \\ Aldric.Degorre, Oded.Maler\}@imag.fr
}

\begin{abstract}
We study a class of scheduling problems which combines the structural aspects associated with task dependencies, with the dynamic aspects associated with ongoing streams of requests that arrive during execution. For this class of problems we develop a scheduling policy which can guarantee bounded accumulation of backlog for all admissible request streams. We show, nevertheless, that no such policy can guarantee bounded latency for all admissible request patterns, unless they admit some laxity.
\end{abstract}

\section{Introduction}

The problem of efficient allocation of reusable resources over time, also known as scheduling, is a universal problem, appearing almost everywhere, ranging from the allocation of machines in a factory $[22,6,19]$, allocation of processor time slots in a real-time system $[20,9]$, allocating communication channels in a network [16], or allocation of vehicles for transportation tasks [7]. Unfortunately, the study of scheduling problems is distributed among many academic communities and application domains, each focusing on certain aspects of the problem.

In the vast scheduling literature, one can, very roughly, identify two generic types of problems. In the first type, the work to be scheduled admits a structure which includes precedence constraints between tasks, but the problems are, more often than not, static: the work to be executed is known in advance and is typically finite. Examples of this type of problems are the job-shop problem motivated by manufacturing (linear precedence constraints, heterogeneous resources) $[19,18]$ or the task-graph scheduling problem, motivated parallel execution of programs (partially-ordered tasks, homogeneous resources) [14] (some recurrent aspects of scheduling are exhibited in program loop parallelization [13] but the nature of uncertainty there is different and rather limited).

On the other hand, in problems related to real-time systems [10] or in queuing theory [17], one is concerned with infinite streams of tasks which arrive either periodically or sporadically (or in a combination of both), satisfying some constraints on task arrival patterns. In many of these "dynamical" problems, the structural dimension of the problem is rather weak, and each request consists of

\footnotetext{
* This research was partially supported by the French Minlogic project ATHole.
} 
a monolithic amount of work. A notable exception is the domain of adversarial queuing theory [8] where some structure and uncertainty are combined.

In this paper we propose a model which combines the dynamic aspect associated with request streams whose exact content is not known in advance, with the structural aspects expressed by task dependencies. We define a scheduling problem where the demand for work is expressed as a stream of requests, each being a structured job taken from a finite set of types, hence such a stream can be viewed as a timed word over the the alphabet of job types. Each job type defines a finite partially-ordered set of tasks, each associated with a resource type and a duration. Such a stream is to be scheduled on an execution platform consisting of a finite number of resources (machines). A schedule is valid relative to a request stream if it satisfies both the precedence constraints imposed by the structure of the jobs and the resource constraints imposed by the number of resources available in the platform (and, of course, it does not execute jobs before they are requested).

The quality of a specific schedule is evaluated according to two types of measures, one associated with the evolution of the backlog over time, that is, the difference between the amount of work requested and the amount of work supplied, and the latency, the temporal distance between the arrival of a job instance and the termination of its execution. To model the uncertain external environment we use the concept of a request generator, a set of request streams satisfying some inter-arrival timing constraints. Such constraints can be expressed, for example, using timed automata [2], real-time logics [3] or timed regular expressions [4]. We restrict the discussion to admissible request streams that do not demand more work over time than the platform can offer. A scheduling policy (strategy) should produce a schedule for each admissible request stream, subject to causality constraints: the decision of the scheduler at a given moment can only be based on the prefix of the request stream it has seen so far.

After defining all these notions we prove two major fundamental results:

- Positive: we develop a scheduling policy which produces a bounded backlog schedule for any admissible request stream. Note that due to the precedence constraints between the tasks in the jobs, request stream admissibility does not, a priori, guarantee the existence of such a schedule. In fact, we show that a naive "oldest first" policy can accumulate an unbounded backlog for certain request streams. Our policy achieves this goal by making decisions that provide for pipelined execution whenever possible.

- Negative: there are admissible request streams for which no bounded-latency schedule (and hence no bounded-latency policy) exists.

The rest of the paper is organized as follows: in Sect. 2 we define our scheduling framework, in Sect. 3 we prove a negative result concerning the impossibility of bounded latency schedules. In Sect. 4 we extend the framework to include scheduling policies and in Sect. 5 we develop a scheduling strategy that guarantees bounded backlog. We conclude with a discussion of past and future work. 


\section{The Recurrent Scheduling Problem}

\subsection{General Definitions}

We use timed words and timed languages to specify streams of requests for work. Intuitively, a timed word such as $\tilde{u}=3 \cdot a_{1} \cdot 2 \cdot a_{2} \cdot a_{3} \cdot 6$ consists of a passage of time of duration 3 , followed by the event $a_{1}$, followed by a time duration 2 , followed by the two events $a_{2}$ and $a_{3}$ and then a time duration of 6 . We present some basic definitions and notations (see more formal details in [4]).

- A word over an event alphabet $\Sigma$ is either $\epsilon$, the empty word, or $u \cdot a$ where $u$ is a word and $a \in \Sigma$. An $\omega$-word is an infinite sequence $\left(a_{i}\right)_{i \in \mathbb{N}} \in \Sigma^{\omega}$.

- A timed word over $\Sigma$ is a word over $\Sigma \cup \mathbb{R}_{+}$. The duration of a timed word $u$, denoted by $|u|$ is the sum of its elements that are taken from $\mathbb{R}_{+}$, for example $|\tilde{u}|=11$. A timed $\omega$-word is an infinite sequence $\left(a_{i}\right)_{i \in \mathbb{N}} \in\left(\Sigma \cup \mathbb{R}_{+}\right)^{\omega}$ such that its duration diverges.

- The concatenation of a word $u$ and a word (or $\omega$-word) $v$ is denoted by $u \cdot v$.

- A word $u$ is a prefix of $v$ iff there exists $w$ such that $v=u \cdot w$, which we denote $u \sqsubseteq v$. We say that $u$ is a proper prefix of $v$, denoted by $u \sqsubset v$, if $u \neq v$.

- A word (or an $\omega$-word) $u$ is a suffix of $v$ iff there exists $w$ such that $v=w \cdot u$.

For a timed $(\omega-)$ word $u$ over $\Sigma$

- By $u(a, i)$ we denote the time of the $i$-th occurrence of event $a \in \Sigma$ in the timed word $u$. Formally $u(a, i)=t$ if $u=v \cdot a \cdot w$ such that $|v|=t$ and $v$ contains $i-1$ occurrences of $a$. We let $u(a, i)=\infty$ when $a$ occurs less than $i$ times in $u$.

- The timed word $u_{[0, t]}$ is the longest prefix of $u$ with duration $t$. Formally $u_{[0, t]}=t_{0} \cdot a_{0} \cdot t_{1} \cdot a_{1} \cdot \ldots \cdot t_{i}$ such that $\sum_{0 \leq k \leq i} t_{k}=t$ and there exists no discrete event $a$ such that $t_{0} \cdot a_{0} \cdot t_{1} \cdot a_{1} \cdot \ldots \cdot t_{i} \cdot a$ is a prefix of $w$. For example, $\tilde{u}_{[0,4]}=3 \cdot a_{1} \cdot 1$ and $\tilde{u}_{[0,5]}=3 \cdot a_{1} \cdot 2 \cdot a_{2} \cdot a_{3} \cdot 0$.

Sets of timed $(\omega$-)words over $\Sigma$ are called timed $(\omega$-)language. We denote the sets of such languages by $\mathcal{T}(\Sigma)$ and $\mathcal{T}_{\omega}(\Sigma)$, respectively.

\subsection{Execution Platform, Jobs and Tasks}

The execution platform determines our capacity to process work.

Definition 1 (Execution Platform) An execution platform over a finite set $M=\left\{m_{1}, \ldots, m_{n}\right\}$ of resource (machine) types is a function $R: M \rightarrow \mathbb{N}$.

Example: $\left\{m_{1} \mapsto 2, m_{2} \mapsto 4, m_{3} \mapsto 1\right\}$ is an execution platform with three resource types $m_{1}, m_{2}, m_{3}$ having 2 instances of $m_{1}, 4$ instances of $m_{2}$, and 1 instance of $m_{3} \cdot{ }^{1}$

\footnotetext{
${ }^{1}$ We will use the notation $R_{m}$ for $R(m)$ and $R$ when we want to treat the whole platform capacity as vector and make component-wise arithmetical operations. The same will hold for sets of functions indexed by the elements of $M$.
} 
The task is the atomic unit of work, specified by the resource type it consumes and by its duration. The job is a unit of a larger granularity, consisting of tasks related by precedence constraints. Each job is an instantiation of a job type.

Definition 2 (Job Type) A job type over a set $M$ of resources is a tuple $J=$ $\langle T, \prec, \mu, d\rangle$ such that $\prec \subseteq T \times T$ and $\langle T, \prec\rangle$ is a finite directed acyclic graph whose nodes are labelled by 2 functions: $\mu: T \rightarrow M$, which associates a task to the resource type it consumes, and $d: T \rightarrow \mathbb{R}_{+}-\{0\}$ specifying task duration.

As an example consider a job type where $T=\left\{a_{1}, a_{2}, a_{3}\right\}, \prec=\left\{\left(a_{1} \prec\right.\right.$ $\left.\left.a_{3}\right),\left(a_{2} \prec a_{3}\right)\right\} \mu=\left\{a_{1} \mapsto m_{1}, a_{2} \mapsto m_{2}, a_{3} \mapsto m_{3}\right\}, d=\left\{a_{1} \mapsto 3, a_{2} \mapsto\right.$ $\left.\left.2, a_{3} \mapsto 1\right\}\right\rangle$, where $a_{1}$ needs resource $m_{1}$ for 3 time units, $a_{2}$ uses resource $m_{2}$ for 2 time units while $a_{3}$ consumes $m_{3}$ for 1 time unit. Task $a_{3}$ cannot start before both $a_{1}$ and $a_{2}$ terminate.

For a set $\mathcal{J}=\left\{\left\langle T_{1}, \prec_{1}, \mu_{1}, d_{1}\right\rangle, \ldots,\left\langle T_{n}, \prec_{n}, \mu_{n}, d_{n}\right\rangle\right\}$ of job types, we let $T_{\mathcal{J}}, \prec \mathcal{J}, \mu_{\mathcal{J}}$ and $d_{\mathcal{J}}$ denote, respectively, the (disjoint) union of $T_{i}, \prec_{i}, \mu_{i}$ and $d_{i}$, for $i=1$..n. We call elements of $T_{\mathcal{J}}$ task types. When $\mathcal{J}$ is clear from the context we use notations $T, \prec, \mu$ and $d$.

Definition 3 (Initial Tasks, Rank) An initial task a is an element of $T$ such that there exists no $a^{\prime} \in T$ with $a^{\prime} \prec a$. The rank of task $a$ is the number of edges of the longest path $a_{0} \prec a_{1} \prec \ldots \prec a$ such that $a_{0}$ is initial. Initial tasks have rank 0 .

\subsection{The Demand}

The sequence of jobs and tasks that should be executed on the platform is determined by a request stream.

Definition 4 (Request Streams and Generators) A request stream over a set $\mathcal{J}$ of job types is a timed $\omega$-word over $\mathcal{J}$. A request generator is a timed $\omega$-language over $\mathcal{J}$.

Each request stream presents a demand for work over time which should not exceed the platform capacity, otherwise the latter will be saturated.

Definition 5 (Work Requested by Jobs and Streams) With each resource type $m$ we define a function $W_{m}: \mathcal{J} \rightarrow \mathbb{R}_{+}$so that $W_{m}(J)$ indicates the total amount of work on $m$ demanded by job $J, W_{m}(J)=\sum_{\left\{a \in T_{J}: \mu(a)=m\right\}} d(a)$. $W e$ lift this function to request stream prefixes by letting $W(\epsilon)=0$, $W(u \cdot t)=W(u)$ for $t \in \mathbb{R}_{+}$and $W(u \cdot J)=W(u)+W(J)$ for $J \in \mathcal{J}$.

We restrict our attention to request streams that do not ask for more work per time unit than the platform can provide, and, furthermore, do not present an unbounded number of requests in a bounded time interval. 
Definition 6 (Admissible, Critical and Subcritical Request Streams) $A$ request stream $\sigma$ is $\alpha$-lax $\left(\alpha \in \mathbb{R}_{+}\right)$with respect to an execution platform $R$ if for every $t<t^{\prime}, W\left(\sigma_{\left[0, t^{\prime}\right]}\right)-W\left(\sigma_{[0, t]}\right) \leq \alpha\left(t^{\prime}-t\right) R+b$ for some constant $b \in \mathbb{R}^{n}$. $A$ stream is admissible if it is $\alpha$-lax for some $\alpha \leq 1$, subcritical if it is $\alpha$-lax for $\alpha<1$ and critical if it is admissible but not subcritical. A request generator $G$ is $\alpha$-lax if every $\sigma \in G$ is $\alpha$-lax.

\subsection{Schedules}

Definition 7 (Schedule) A schedule is a function $s: T \times \mathbb{N} \rightarrow \mathbb{R}_{+}^{\infty}$ (where $\mathbb{R}_{+}^{\infty}=\mathbb{R}_{+} \cup\{\infty\}$ with the usual extension of the order and operations).

The intended meaning of $s(a, i)=t$ is that the $i$-th instance of task $a$ (which is part of the $i$-th instance of the job type to which it belongs) starts executing at time $t$. If we restrict ourselves to "non-overtaking" schedules ${ }^{2}$ such that $s(a, i) \leq$ $s\left(a, i^{\prime}\right)$ whenever $i<i^{\prime}$, we can view a schedule as a timed $\omega$-word in $\mathcal{T}_{\omega}(T)$. Likewise we can speak of finite prefixes $s_{[0, t]}$ which are timed words in $\mathcal{T}(T)$.

Since tasks have fixed durations and cannot be preempted, a schedule determines uniquely which tasks are executed at any point in time and, hence, how many resources of each type are utilized, a notion formalized below.

Definition 8 (Utilization Function, Work Supplied) The resource utilization function associated with every resource $m$ is $U_{m}: \mathcal{T}_{\omega}(T) \times \mathbb{R}_{+} \rightarrow \mathbb{N}$ defined as $U_{m}(s, t)=|\{(a, i) \in T \times \mathbb{N}: \mu(a)=m \wedge s(a, i) \leq t<s(a, i)+d(a)\}|$. The work supplied by a prefix of $s$ is the accumulated utilization: $W\left(s_{[0, t]}\right)=\int_{0}^{t} U(s, \tau) d \tau$.

Definition 9 (Valid Schedule) A schedule $s$ is valid for a request stream $\sigma$ on an execution platform $R$ if for any task instance $(a, i)$

- if $J$ is the job type a belongs to, then $s(a, i) \geq \sigma(J, i)$ (no proactivity: jobs are executed after they are requested);

- $\forall a^{\prime}, a^{\prime} \prec a, s(a, i) \geq s\left(a^{\prime}, i\right)+d\left(a^{\prime}\right)$ (job precedences are met);

- $\forall t \in \mathbb{R}_{+}, U(s, t) \leq R$ (no overload: no more resource instances of a type are used than their total amount in the execution platform).

The quality of a schedule can be evaluated in two principal and related (but not equivalent) ways, the first of which does not look at individual job instances but is based on the amount of work. During every prefix of the schedule there is a non-negative difference between the amount of work that has been requested and the amount of work that has been supplied. This difference can be defined in a "continuous" fashion like $\Delta_{\sigma, s}(t)=W\left(\sigma_{[0, t]}\right)-W\left(s_{[0, t]}\right)$. An alternative that we will use, is based on the concept of residue or backlog, which is simply the set of requested tasks that have not yet started executing. It is not hard to see that a bounded residue is equivalent to a bounded difference between requested and supplied work.

\footnotetext{
${ }^{2}$ Note that non-overtaking applies only to tasks of the same type.
} 
Definition 10 (Residue, Bounded Residue Schedules) The residue associated with a request stream $\sigma$ and a valid schedule $s$ at time $t$ is $\rho_{\sigma, s}(t)=$ $\{(a, i) \in T \times \mathbb{N}: \sigma(a, i) \leq t<s(a, i)\}$. A valid schedule $s$ is of bounded residue if there is a number $c$ such that $\left|\rho_{\sigma, s}(t)\right| \leq c$ for every $t$.

The second performance measure associated with a schedule is related to latency, the time an individual job has to wait between being requested and the completion time of its last task.

Definition 11 (Latency) Given a request stream $\sigma$ and a valid schedule $s$, the latency of a job instance $(J, i)$ is $L_{J, i}(\sigma, s)=\max _{a \in T_{J}}\{(s(a, i)+d(a))\}-\sigma(J, i)$. The latency of $s$ with respect to $\sigma$ is $L(\sigma, s)=\sup _{J \in \mathcal{J}, i \in \mathbb{N}} L_{J, i}(\sigma, s)$.

Note that it is possible that every job instance is served in finite time but the latency of the schedule is, however, infinite, that is, the sequence $\left\{L_{J, i}\right\}_{i \in \mathbb{N}}$ may diverge. Bounded residue does not imply bounded latency: we can keep one job waiting forever, while still serving all the others without accumulating backlog. But the implication holds in the other direction.

Lemma 1 A valid schedule with bounded latency has a bounded residue.

Proof. Let $s$ be a valid schedule with latency $\lambda \in \mathbb{R}_{+}$. Let $V(t)$ be the total amount of work of the tasks that are in the residue at time $t$. Since all these tasks are supposed to be completed by $t+\lambda$ we have $V(t) \leq \lambda R$ which implies a bound on the residue.

\subsection{The Running Example}

We will use the following recurrent scheduling problem to construct the negative result and to illustrate our scheduling policy. Consider a platform over $M=$ $\left\{m_{1}, m_{2}\right\}$ with $R\left(m_{1}\right)=R\left(m_{2}\right)=1$. The set of job types is $\mathcal{J}=\{A, B\}$ whose respective sets of tasks $\left\{a_{1} \prec a_{2}\right\}$ and $\left\{b_{1} \prec b_{2}\right\}$ have all a unit duration. The difference between these job types is that $A$ uses $m_{1}$ before $m_{2}$ while $B$ uses $m_{2}$ before $m_{1}$ (see Fig. 1). As a request generator we consider $G=((A \cdot 1)+(B \cdot 1))^{\omega}$, that is, every unit of time, an instance of either one of these jobs is requested (to simplify notations we will use henceforth $A$ and $B$ as a shorthand for $A \cdot 1$ and $B \cdot 1$, respectively). Since each job type requires exactly the amount of work offered by the platform, $G$ is admissible and, in fact, critical. A bounded-residue schedule for such critical request streams should keep the machines busy all the time except for some intervals (that we call utilization gaps) whose sum of durations is bounded.

The reversed order of resource utilization in $A$ and $B$ renders these two job types incompatible in the sense that it is not easy to "pipeline" them on our platform. Intuitively at the moment a request stream switches from $A$ to $B$, we may have tasks $a_{2}$ and $b_{1}$ ready for execution but only one instance of their common resource $m_{2}$ is free. Our scheduling policy will, nevertheless, manage to pipeline them but, as we show in the next section, bounded latency schedules are impossible. 


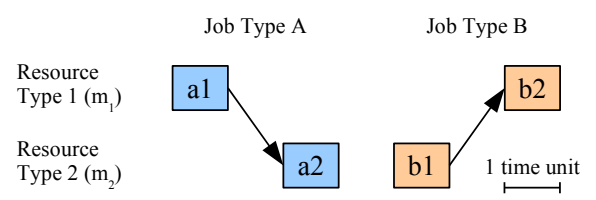

Fig. 1. The example.

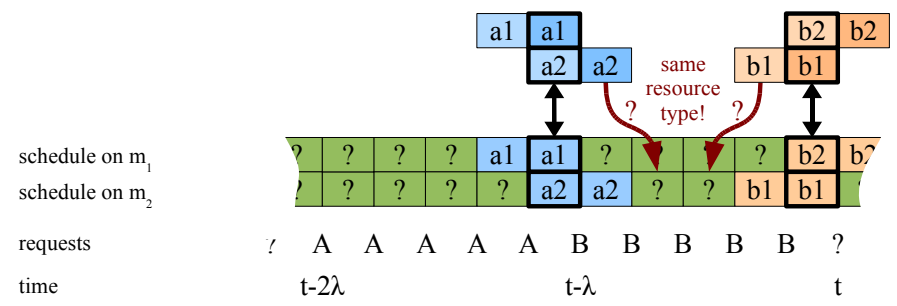

Fig. 2. An illustration of the fact that a request segment $A^{\lambda} \cdot B^{\lambda}$ implies a utilization gap in any schedule of latency $\lambda$ or less. Before $t-\lambda$ : job type $A$ has been requested for a long time, so the residue contains only tasks from $A$. At $t-\lambda$ : from now on, requests are of type $B$. After $t-1$ : if the latency is $\lambda$, there should be no more tasks from $A$ in the residue. Now between $t-\lambda$ and $t-1$, only suffixes of $A$ and prefixes of $B$ can be scheduled, and among those, at least one proper suffix.

\section{$3 \quad$ Negative Result}

Theorem 1 Some admissible request streams admit no bounded-latency schedule.

We prove the theorem using the following lemma, which shows that for any latency $\lambda$, the occurrence of a certain request pattern implies a unit increase in the residue and hence infinitely many consecutive repetitions of this pattern will imply an unbounded residue. The statement of the lemma and its proof are illustrated in Fig. 2.

Lemma 2 Let $\sigma$ be a request stream with a prefix of the form $\sigma_{[0, t]}=u \cdot A^{\lambda} \cdot B^{\lambda}$ and let $s$ be a valid schedule for $\sigma$ with latency $\lambda$. Then there is a utilization gap (an idle resource) of duration 1 or more in the interval $[t-\lambda-1, t]$.

Proof. Since the latency of $s$ is $\lambda$, no task instance of $B$ belongs to the residue $\rho_{\sigma, s}(t-\lambda-1)$, so the only way to avoid a gap at time $t-\lambda-1$ is to schedule an instance of $a_{1}$ and an instance of $a_{2}$. For the same reason, $\rho_{\sigma, s}(t-1)$ contains no task instance of $A$, so that at time $t-1, s$ schedules $b_{1}$ and $b_{2}$. Moreover any task instance of $B$ in the residue after $t-\lambda-1$ is an instance that was requested since $t-\lambda$.

Now what happens in $[t-\lambda, t-1]$ ? In that interval, the residue has task instances from requests for $A$ made before $t-\lambda$ and from requests for $B$ made 
since that time. Due to bounded latency all the instances from $A$ are due for $t-1$. We also know that, because $a_{1} \prec a_{2}$, the residue has always more $a_{2}$ than $a_{1}$, and that their amount is the same only when all started job instances of $A$ are finished, which is not possible at $t-1$ because an $a_{1}$ is scheduled for $t-\lambda-1$ (and thus task $a_{2}$ of the same job instance cannot start before $t-\lambda$ ). In that interval we also schedule task instances from $B$ the earliest of which can have started execution at $t-\lambda$. Thus, since $b_{1} \prec b_{2}$, we cannot schedule more $b_{2}$ than $b_{1}$.

Summing up the quantity of work scheduled by $s$ between $t-\lambda$ and $t$, we find that on $m_{1}$ we schedule $n_{a_{1}}$ instances of $a_{1}$ and $n_{b_{2}}$ instances of $b_{2}$ and on $m_{2}$ we schedule $n_{a_{2}}$ instances of $a_{2}$ and $n_{b_{1}}$ instances of $b_{1}$, satisfying $n_{a_{2}}>n_{a_{1}}$ and $n_{b_{1}} \geq n_{b_{2}}$. Thus $m_{2}$ performs at least one unit of work more than $m_{1}$ in the same interval, which is only possible if $m_{1}$ admits a utilization gap of duration 1.

Consider now a request stream that has infinitely many occurrences of the pattern $u \cdot A^{\lambda} \cdot B^{\lambda}$. A schedule with latency $\lambda$ for this stream will have infinitely many gaps, and hence an unbounded residue, a fact which contradicts Lemma 1. Hence such a stream admits no schedule whose latency is $\lambda$ or less.

Proof (of Theorem 1). Consider now any request stream $\sigma$ in the language $L_{\infty}=$ $\mathcal{J}^{*} \cdot A \cdot B \cdot B \cdot \mathcal{J}^{*} \cdot A \cdot A \cdot A \cdot B \cdot B \cdot B \cdot B \cdot \mathcal{J}^{*} \cdot A \cdot A \cdot A \cdot B \cdot B \cdot B \cdot \cdot$, where $\mathcal{J}$ stands for $(A+B)$. For every $\lambda, \sigma$ has infinitely many prefixes of the form $u \cdot A^{\lambda} \cdot B^{\lambda}$ and cannot have a schedule of latency $\lambda$. Consequently it admits no bounded latency schedule.

Note that this impossibility result is not related to the dynamic aspect of the scheduling problem. Even a clairvoyant scheduler who knows the whole request stream in advance cannot find a bounded latency solution.

Note also that the language $L_{\infty}$ is not pathological. If fact, in any reasonable way to induce probabilities on $(A+B)^{\omega}$, this language will have probability of 1 . Hence we can say that critical systems having two incompatible jobs will almost surely admit only unbounded-latency schedules.

\section{Scheduling Policies}

Now we want to consider the act of scheduling as a dynamic process where a scheduler has to adapt its decisions to the evolution of the environment, here the incoming request stream. We want the scheduler to construct a schedule incrementally as requests arrive. The mathematical object that models the procedure of mapping request stream prefixes into scheduling decisions is called a scheduling policy or a strategy.

Formally speaking, a policy can be viewed as a timed transducer, a causal function $p: \mathcal{T}_{\omega}(\mathcal{J}) \rightarrow \mathcal{T}_{\omega}(T)$ which produces for each request stream $\sigma$ a valid schedule $s=p(\sigma)$. Causality here means that the value of $s_{[0, t]}$ depends only on $\sigma_{[0, t]}$. We will represent the policy as a procedure $p$ which, at each time instant $t$, looks at $\sigma_{[0, t]}$ and selects a (possibly empty) set of task instances to 
be scheduled for execution at time $t$, that is, $s(a, i)=t$ if $(a, i) \in p\left(\sigma_{[0, t]}\right)$. We will use $s_{[0, t]}=p\left(\sigma_{[0, t]}\right)$ to denote the schedule prefix constructed by successive applications of $p$ during the interval $[0, t]$. We assume that each policy is designed to work with admissible request streams taken from a generator $G \subseteq \mathcal{T}_{\omega}(\mathcal{J})$.

Definition 12 (Scheduling policy) A scheduling policy is a function $p: \mathcal{T}(\mathcal{J}) \rightarrow 2^{T \times \mathbb{N}}$ such that for every task instance $(a, i)$ and a request stream prefix $\sigma,(a, i) \in p(\sigma)$ implies that $(a, i) \notin p\left(\sigma^{\prime}\right)$ for any $\sigma^{\prime} \sqsubset \sigma$. A scheduling pol$i c y$ is valid for $\sigma$ if for every $t$, the obtained schedule $s_{[0, t]}=p\left(\sigma_{[0, t]}\right)$ satisfies the conditions of Definition 9, namely, no proactivity and adherence to precedence and resource constraints.

We evaluate the overall performance of a policy based on the worst schedule it produces over the streams in the generator. Since we have just shown a negative result concerning latencies, we focus on the residue.

Definition 13 (Bounded Residue Policies) A scheduling policy has a bounded residue relative to a generator $G$ if it produces a bounded-residue schedule for every $\sigma \in G$.

In the following, we use notation $\rho_{\sigma, p}$ instead of $\rho_{\sigma, p(\sigma)}$ to denote the residue resulting from the application of a policy $p$ to a request stream (or prefix) $\sigma$.

\section{Positive Result}

In this section we show that any recurrent scheduling problem with an admissible request generator admits a policy in the sense of Sect. 4 which maintains the residue bounded. We emphasize again that the policy makes decisions at run time without knowing future requests.

\subsection{Oldest-First Policy Does Not Work}

To appreciate the difficulty, let us consider first a naive Oldest-First policy: whenever the number of tasks that are ready to use a resource is larger than the number of free instances of the resource, the available instances are granted to the older tasks among them. We show that this policy fails to guarantee bounded residues.

Theorem 2 The Oldest-First policy cannot guarantee a bounded residue.

In fact, this policy will lead to an unbounded residue schedule for request streams in the language $L_{\infty}$ of the previous section as illustrated in Fig. 3 and proved below. The reason is, again, the incompatibility between the job types, which leads to infinitely many utilization gaps where a resource is free while none of the corresponding tasks in the residue is ready to utilize it. The result is a direct corollary of the following lemma: 
Lemma 3 A bounded residue schedule which conforms to the Oldest-First policy has a bounded latency.

Note that we already proved the converse for arbitrary schedules and policies.

Proof. First we show that any task instance $(a, i)$ that becomes eligible for execution at time $t$, is scheduled for execution within a bounded amount of time after $t$. This holds because, following the policy, the only tasks that can be executed between $t$ and $s(a, i)$ are those that are already in the (bounded) residue at time $t$. Next we show, by induction on the rank of the tasks, that this fact implies that any task is executed within a bounded amount of time after its job is issued. This holds trivially for the initial tasks which become eligible for execution immediately when the job arrives and then holds for tasks of rank $n+1$ by virtue of the bounded latency of tasks of rank $n$. Thus the latency of a bounded-residue schedule produced by the Oldest-First has to be bounded.

Since we have already shown that request streams in $L_{\infty}$ do not admit bounded-latency schedules, a bounded residue strategy will lead to a contradiction and this proves Theorem 2. Like the case for Theorem 1, under reasonable probability assignments to jobs, one can show that the Oldest-First policy will almost surely lead to unbounded-residue schedules when applied to critical streams of incompatible jobs.

\begin{tabular}{|c|c|c|c|c|c|c|c|c|c|c|c|c|c|}
\hline schedule on $\mathrm{m}_{1}$ & a1 & & & b2 & b2 & a1 & al & a1 & & & b2 & $\mathrm{b} 2$ & b2 \\
\hline schedule on $\mathrm{m}_{2}$ & & $\mathrm{a} 2$ & b1 & b1 & & & a2 & $\mathrm{a} 2$ & $\mathrm{a} 2$ & b1 & b1 & b1 & b1 \\
\hline residue on $\mathrm{m}_{1}$ & 0 & 1 & 2 & 2 & 2 & 2 & 2 & 2 & 3 & 4 & 4 & 4 & 4 \\
\hline residue on $\mathrm{m}_{2}$ & 1 & 1 & 1 & 1 & 2 & 3 & 3 & 3 & 3 & 3 & 3 & 3 & 3 \\
\hline
\end{tabular}

Fig. 3. Schedule generated by the "oldest first" policy on a stream in the language $L_{\infty}$, described in 3 . Here we see that a gap of length 2 is created on one of the resource types at every change of job type in the request stream, which makes the residue grow indefinitely.

\subsection{A Bounded Residue Policy}

Theorem 3 Any admissible generator admits a bounded-residue scheduling pol$i c y$.

In order to circumvent the shortcomings of the "Oldest First" policy, we describe in the sequel a policy that eventually reaches the following situation: whenever a resource becomes free and the residue contains tasks that need it, at least one of those tasks will be ready for execution. 
The policy is described in detail in Algorithm 1 and its proof is omitted due to space limitations. We explain the underlying intuition below. The policy separates the act of choosing which tasks to execute in the future from the act of actually starting them. The first decision is made upon job arrival while the second is made whenever a resource is free and a corresponding task has been selected. To this end we partition the residue into two parts. The first part $P$ (the "pool") consists of requested task instances that have not yet been selected for execution. Among those, only task instances whose $\prec$-predecessors have already terminated are eligible for being selected and moved to the other part, which consists of $n$ FIFO queues $\left\{Q_{m}\right\}_{m \in M}$, one for each resource type. The passage between the two is controlled by two types of events:

- Task termination: when a task $(a, i)$ terminates, eligibility status of its successors in $P$ is updated;

- Job arrival: when a job instance $(J, i)$ arrives we pick the oldest ${ }^{3}$ eligible instance $\left(a, j_{a}\right) \in P$ (if such exists) for every task type $a \in T_{J}$ such that $\mu(a)=m$, and move it to $Q_{m}$. Note that only initial tasks of $(J, i)$ are eligible for being selected when $(J, i)$ arrives, while for other task types only earlier instances can be chosen.

Whenever a resource of type $m$ is free and $Q_{m}$ is not empty, the first element is removed from $Q_{m}$ and starts executing. This is sufficient to ensure a bounded residue. However, to improve the performance of the algorithm when the streams are subcritical, we also choose to start the oldest eligible task which requires $m$ if an instance of $m$ is released when $Q_{m}$ is empty.

The intuition why this policy works is easier to understand when we look at critical request streams. For such streams, any job type which is requested often enough will eventually have instances of each of its tasks in $Q$ and hence, whenever a resource is freed, there will always be some task ready to use it. This guarantees smooth pipelining and bounded residue for all admissible request streams. In Fig. 4 we can see how our policy schedules the request stream $\sigma_{\infty}=$ $A \cdot B \cdot B \cdot A \cdot A \cdot A \cdots$.

Scheduling policies of the FIFO type have also been studied in the context of adversarial queuing and it has been shown under various hypothesis [12] that those were not stable, sometimes even for arbitrarily small loads. What makes our policy work is the fact that the act of queuing is triggered by a global event (arrival of a new job request) on which the actual choice of tasks to be queued depends. So the decision is somehow "conscious" of the global state of the system, as opposed to what happens in a classical FIFO network.

\subsection{Bounded Latency for Subcritical Streams}

We just showed that a policy could ensure bounded residues in the case of critical streams for which one needs full utilization. But criticality is just a limit case and for that reason it is interesting to know whether such a policy can adapt and

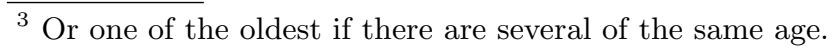




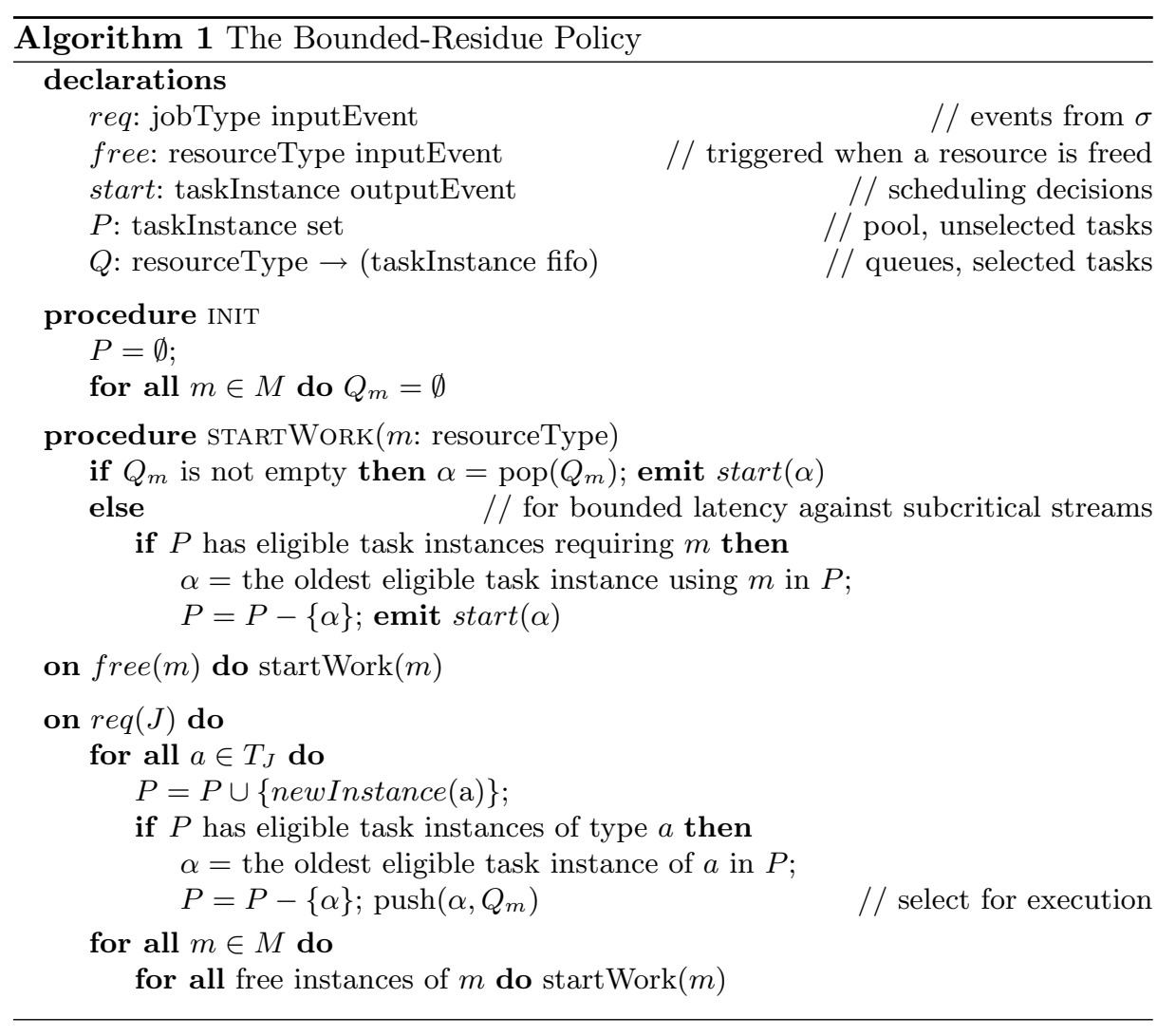

\begin{tabular}{|c|c|c|c|c|c|c|c|c|c|c|c|c|c|}
\hline \multirow{2}{*}{$\begin{array}{l}\text { schedule on } \mathrm{m}_{1} \\
\text { schedule on } \mathrm{m}_{2}\end{array}$} & a1 & & b2 & a1 & a1 & a1 & b2 & b2 & b2 & b2 & a1 & a1 & al \\
\hline & & b1 & b1 & a2 & $\mathrm{a} 2$ & a2 & b1 & b1 & b1 & b1 & $\mathrm{a} 2$ & a2 & $\mathrm{a} 2$ \\
\hline \multirow{2}{*}{$\begin{array}{l}\text { residue on } \mathrm{m}_{1} \\
\text { residue on } \mathrm{m}_{2}\end{array}$} & 0 & 1 & 1 & 1 & 1 & 1 & 1 & 1 & 1 & 1 & 1 & 1 & 1 \\
\hline & 1 & 1 & 1 & 1 & 1 & 1 & 1 & 1 & 1 & 1 & 1 & 1 & 1 \\
\hline
\end{tabular}

Fig. 4. The schedule generated by the bounded-residue policy for $\sigma_{\infty}$. We can see that after the arrival of the second $B$, every resource is always occupied, and that the residue does not grow after that. 
behave better when the request stream is subcritical. Fortunately the answer is positive: the previously exhibited policy, by starting tasks which are not queued when a resource would be otherwise idle, ensures bounded latencies for request streams that admit some laxity.

Theorem 4 The policy described by Algorithm 1 has a bounded latency when applied to any $\alpha$-lax stream with $\alpha<1$.

Lemma 4 There exists a time bound $T_{\alpha, m}$ such that any interval $\left[t, t+T_{\alpha, m}\right]$ admits a time instant where $Q_{m}$ is empty, an instance of $m$ is free and no new request arrives.

Sketch of proof. Consider an interval of the form $[t, t+d]$ in which no machine of type $m$ is idle. The quantity of work dequeued from $Q_{m}$ is $R_{m} d$ and, due to laxity, the amount of work enqueued into $Q_{m}$ is at most $(1-\alpha) R_{m} d$. Hence the total contribution to the amount of work in $Q_{m}$ is $(\alpha-1) R_{m} d$ and for some sufficiently large $d$ it will empty $Q_{m}$.

Proof (of Theorem 4). We know that, when a task in the pool becomes the oldest task of the residue which is not queued, it becomes eligible in a bounded amount of time (all its predecessors must be in the queue). Thus we know that at most $T_{\alpha, m}$ units of time after that, this task is started (either queued or started to fill a gap). Since furthermore the residue (and hence the pool) is bounded (Thm. 3), there is a bound on the time it takes a task to become the oldest in the pool and hence to be executed. Thus we conclude that the latency of the policy is bounded.

\section{Discussion}

We have proved some fundamental results on a model that captures, we believe, many real-world phenomena. Let us mention some related attempts to treat similar problems. The idea that verification-inspired techniques can be used to model and then solve scheduling problems that are not easy to express in traditional real-time scheduling models has been studied within the timed controller synthesis framework and applied to scheduling problems $[23,21,5,1]$. What is common to all these approaches (including [15] which analyzes given policies that admit task preemption) is that the scheduler is computed using a verification/synthesis algorithm for timed automata, which despite several improvements [11] are intrinsically not scalable. The policy presented in this paper does not suffer from this problem, it only needs the request generator to be admissible. Explicit synthesis may still be needed in more complex settings.

In the future it would be interesting to investigate various extensions of the model and variations on the rules of the game, in particular, moving from worstcase reasoning to average case by using probabilistic request generators and evaluating policies according to expected backlog or latency. Finally, we intend to look closer at the question of "pipelinability", that is, the mutual compatibility 
of a set of job types. Results in this direction may lead to new design principles for request servers.

Acknowlegments: We thank anonymous referees for their comments. We are indebted to Viktor Schuppan for his great help in writing the paper.

\section{References}

1. K. Altisen, G. Gößler, A. Pnueli, J. Sifakis, S. Tripakis, and S. Yovine. A framework for scheduler synthesis. In IEEE Real-Time Systems Symposium, pages 154-163, 1999.

2. R. Alur and D. Dill. A theory of timed automata. Theor. Comput. Sci., 126(2):183235, 1994

3. R. Alur and T. Henzinger. Logics and models of real time: A survey. In REX Workshop, pages 74-106, 1991.

4. E. Asarin, P. Caspi, and O. Maler. Timed regular expressions. J. ACM, 49(2):172206,2002

5. E. Asarin, O. Maler, and A. Pnueli. Symbolic controller synthesis for discrete and timed systems. In Hybrid Systems II, pages 1-20, 1994.

6. J. Blazewicz, K. H. Ecker, E. Pesch, G. Schmidth, and J. Weglarz. Scheduling Computer and Manufacturing Processes. Springer, 2nd edition, 2001.

7. L. Bodin, B. Golden, A. Assad, and M. Ball. Routing and scheduling of vehicles and crews : The state of the art. Computers $\& 5$ OR, 10(2):63-211, 1983.

8. A. Borodin, J. Kleinberg, P. Raghavan, M. Sudan, and D. P. Williamson. Adversarial queuing theory. J. ACM, 48(1):13-38, 2001.

9. G. Bottazzo. Hard Real-Time Computing Systems: Predictable Schedulding Algorithms and Applications. Real-Time Systems Series. Springer, 2nd edition, 2005.

10. M. Caccamo, T. Baker, A. Burns, G. Buttazzo, and L. Sha. Real-time scheduling for embedded systems. In D. Hristu-Varsakelis and W. Levine, editors, Handbook of Networked and Embedded Control Systems, pages 173-196. Birkhäuser, 2005.

11. F. Cassez, A. David, E. Fleury, K. Larsen, and D. Lime. Efficient on-the-fly algorithms for the analysis of timed games. In CONCUR, pages 66-80, 2005.

12. V. Cholvi and J. Echagüe. Stability of fifo networks under adversarial models: State of the art. Computer Networks, 51(15):4460-4474, 2007.

13. A. Darte, Y. Robert, and F. Vivien. Scheduling and Automatic Parallelization. Birkhauser Boston, 2000.

14. H. El-Rewini. Partitioning and scheduling. In A. Zomaya, editor, Parallel $\mathcal{6}$ Distributed Computed Handbook, chapter 9, pages 239-273. McGraw-Hill, 1996.

15. E. Fersman, L. Mokrushin, P. Pettersson, and W. Yi. Schedulability analysis of fixed-priority systems using timed automata. Theor. Comput. Sci., 354(2):301-317, 2006.

16. C.-H. Gan, P. Lin, N.-C. Perng, T.-W. Kuo, and C.-C. Hsu. Scheduling for timedivision based shared channel allocation for UMTS. Wirel. Netw., 13(2):189-202, 2007.

17. G.-H. Hsu. A survey of queueing theory. Ann. Oper. Res., 24(1-4):29-43, 1990.

18. A. Jain and S. Meeran. A state-of-the-art review of job-shop scheduling techniques, 1998.

19. E.G. Coffman Jr., editor. Computer and Job-Shop Scheduling Theory. J. Wiley, New York, 1976. 
20. C. Liu and J. Layland. Scheduling algorithms for multiprogramming in a hardreal-time environment. J. ACM, 20(1):46-61, 1973.

21. O. Maler, A. Pnueli, and J. Sifakis. On the synthesis of discrete controllers for timed systems (an extended abstract). In STACS, pages 229-242, 1995.

22. M. Pinedo. Planning and Scheduling in Manufacturing and Services. Springer Series in Operations Research and Financial Engineering. Springer, 2007.

23. H. Wong-Toi and D. Dill. Synthesizing processes and schedulers from temporal specifications. In $C A V$, pages $272-281,1990$. 